\title{
Serum chemerin in patients with rheumatoid arthritis: can it unravel the Chimera of concomitant type 2 diabetes?
}

\author{
Dimitrios loannis Patoulias
}

First Department of Internal Medicine, Hippokration General Hospital, Thessaloniki, Greece

\section{Dear Editor,}

I read with great interest the results of the recently published case-control study conducted by Mohammed Ali et al., enrolling subjects with rheumatoid arthritis (RA) and assessing the diagnostic implications of serum chemerin and visfatin [1]. The authors demonstrated that serum chemerin exhibits good sensitivity and specificity for the diagnosis of RA (sensitivity $=88.1 \%$, specificity $=75.9 \%$, for a cut-off value of $187.88 \mathrm{ng} / \mathrm{ml}$ ), while it significantly correlates with duration of RA, disease activity and major lipid parameters, reflecting an association with systemic inflammation and potentially with atherosclerosis [1].

Former studies have documented that chemerin might be a predictor of type 2 diabetes mellitus (T2DM) development, after adjustment for significant confounding variables [2, 3]. In addition, recent data suggest that among patients with established T2DM diagnosis, chemerin shows a significant positive correlation with body mass index and insulin resistance [4]. Other studies have provided insights into the significant, positive correlation between circulating chemerin levels and either microvascular complications or subclinical atherosclerosis in patients with T2DM, adding to its prognostic value [5-7].

Since RA patients have an increased risk for developing T2DM [8] and have comparable risk for cardiovascular disease development with diabetic subjects [9], it would be interesting to know whether serum chemerin levels in the cohort of Mohammed Ali et al. correlated with T2DM status at baseline or even with glycemic indices, such as fasting plasma glucose, especially after noting that mean body mass index classified the enrolled subjects as overweight $\left(28.11 \pm 2.27 \mathrm{~kg} / \mathrm{m}^{2}\right)$.

Identification of a positive association between serum chemerin levels and diabetes status or glycemic indices among patients with RA could provide further diagnostic and prognostic implications in clinical practice, in terms of risk stratification and treatment strategy in this sensitive population.

The author declares no conflict of interest.

\section{References}

1. Mohammed Ali DM, Al-Fadhel SZ, Abdulsada Al-Ghuraibawi NH, Al-Hakeim HK. Serum chemerin and visfatin levels and their ratio as possible diagnostic parameters of rheumatoid arthritis. Reumatologia 2020; 58: 67-75, DOI: 10.5114/ reum.2020.95359.

2. Bobbert T, Schwarz F, Fischer-Rosinsky A, et al. Chemerin and prediction of Diabetes mellitus type 2. Clin Endocrinol (Oxford) 2015; 82: 838-843.

3. Derosa G, Catena G, Gaudio G, et al. Adipose tissue dysfunction and metabolic disorders: Is it possible to predict who will develop type 2 diabetes mellitus? Role of markErs in the progreSsion of dlabetes in obese paTleNts (The RESISTIN trial). Cytokine 2020; 127: 154947, DOI: 10.1016/j.cyto.2019.154947.

4. Akgul Balaban Y, Yilmaz N, Kalayci M, et al. Irisin and chemerin levels in patients with type 2 diabetes mellitus. Acta Endocrinol (Buchar) 2019; 15: 442-446, DOI: 10.4183/aeb.2019.442.

5. Lachine NA, Elnekiedy AA, Megallaa MH, et al. Serum chemerin and high-sensitivity $C$ reactive protein as markers of subclinical atherosclerosis in Egyptian patients with type 2 diabetes. Ther Adv Endocrinol Metab 2016; 7: 47-56, DOI: 10.1177/2042018816637312.

6. Gu P, Wang W, Yao Y, et al. Increased Circulating Chemerin in Relation to Chronic Microvascular Complications in Patients with Type 2 Diabetes. Int J Endocrinol 2019; 2019: ID 8693516, DOI: 10.1155/2019/8693516.

7. Yasir M, Senthilkumar GP, Jayashree K, et al. Association of serum omentin-1, apelin and chemerin concentrations with the presence and severity of diabetic retinopathy in type 2 diabetes mellitus patients. Arch Physiol Biochem 2019 [published online ahead of print], DOI: 10.1080/13813455.2019.1680698.

8. Jiang P, Li H, Li X. Diabetes mellitus risk factors in rheumatoid arthritis: a systematic review and meta-analysis. Clin Exp Rheumatol 2015; 33: 115-121.

9. Peters MJ, van Halm VP, Voskuyl AE, et al. Does rheumatoid arthritis equal diabetes mellitus as an independent risk factor for cardiovascular disease? A prospective study. Arthritis Rheum 2009; 61: 1571-1579, DOI: 10.1002/art.24836.

\footnotetext{
Address for correspondence:

Dimitrios Ioannis Patoulias, First Department of Internal Medicine, Hippokration General Hospital, 49 Konstantinoupoleos St.,

Thessaloniki 54642 Greece, e-mail: dipatoulias@gmail.com

Submitted: 8.06.2020; Accepted: 30.06.2020
} 